\title{
Enhanced Quantification for 3D Energy Dispersive Spectrometry: Going Beyond the Limitation of Large Volume of X-Ray Emission
}

\author{
Pierre Burdet, ${ }^{1, *}$ Cécile Hébert, ${ }^{2}$ and Marco Cantoni ${ }^{2}$ \\ ${ }^{1}$ Department of Materials Science and Metallurgy, University of Cambridge, Charles Babbage Road 27, Cambridge CB3 OFS, \\ Cambridgeshire, UK \\ ${ }^{2}$ Centre Interdisciplinaire de Microscopie Electronique, Ecole Polytechnique Fédérale de Lausanne (EPFL), Station 12, Lausanne \\ 1015, Switzerland
}

\begin{abstract}
This paper presents a method developed to quantify three-dimensional energy dispersive spectrometry (3D EDS) data with voxel size smaller than the volume from which X-rays are emitted. The influence of the neighboring voxels is corrected by applying recursively a complex quantification, improving thereby the accuracy of the quantification of critically small features. The enhanced quantification method is applied to simulated and measured data. A systematic improvement is obtained compared with classical quantification, proving the concept and the prospect of this method.
\end{abstract}

Key words: energy dispersive $\mathrm{X}$-ray spectrometry, focused ion beam, tomographic spectral imaging, 3D chemical analysis, 3D microanalysis, quantification, 3D image analysis

\section{INTRODUCTION}

Capabilities of the scanning electron microscope (SEM) are extended to elemental analysis with energy dispersive spectrometry (EDS). EDS analysis is performed at the electron-beam position and is used to determine the local composition of the specimen. A main limitation of this technique is due to the electron-beam energy that needs to be high enough to efficiently excite the characteristic X-ray lines of interest. The high energy electrons consequently spread deep into the material, and the volume from which X-rays are emitted is large, roughly in the micrometer range under typical conditions. Such a large volume of emission affects the analysis as illustrated in Figure 1 with an exemplary sample formed by a particle (light gray) in a matrix (dark gray). The particle is smaller than the volume of primary generated X-rays drawn with a black outline. Some of the X-rays are emitted from the feature's surrounding. The recorded spectrum is only partially characteristic for the particle. The large volume of X-ray emission is hence the main factor limiting the smallest size of features that can be directly analyzed quantitatively by SEM/EDS spectrometry.

Despite this limitation, the SEM/EDS instrument is widely used for elemental mapping as reviewed by Friel \& Lyman (2006). Using a dual-beam microscope formed by a SEM equipped with a focused ion beam (FIB), EDS mapping can be extended to three-dimensional (3D) EDS microanalysis. The experimental method used in this work was developed by Schaffer et al. (2007). It is illustrated

Received September 24, 2013; accepted May 29, 2014

*Corresponding author.pb565@cam.ac.uk in Figure 2. Before acquisition, the sample area to be analyzed is prepared: the sample is tilted perpendicularly to the ion beam and trenches are milled away around the volume of interest. The freshly milled cross-section surrounded by a dashed stroke in Figure 2 is analyzed during acquisition. This surface has a tilt angle that is complementary to the angle between the two columns, typically $36^{\circ}$. In the sequential acquisition, thin layers of material are milled away by the ion beam through the volume of interest, and each section is characterized by SEM imaging and EDS mapping. The recorded 3D data set is thus composed of a stack of SEM images and a stack of EDS maps which are spectral images.

The complex acquisition presented in Figure 2 induces three specific limitations. (1) The analyzed surface is surrounded by trenches, in which spurious X-rays are generated and can be detected, as reported by Schaffer \& Wagner (2008). (2) As the acquisition lasts longer, global conditions of the whole system, such as temperature, are more likely to vary inducing mechanical drift that can affect the milling operations or image acquisition. (3) The time per spectrum is reduced in order to be able to characterize a large volume in a reasonable total acquisition time. The obtained spectra suffer from low total number of X-ray counts leading to high noise. Since Schaffer's work, faster detectors such as the silicon drift detector, have become available. This greatly improves the counting statistics. 3D EDS microanalysis has become significantly faster on the experimental side.

The procedure to determine the local sample composition from a spectrum is referred to as quantification. Classical quantification methods assume the sample to be homogeneous over the full range of X-ray generation (Goldstein et al., 2003, chap. 9). Some methods were extended to heterogeneous 


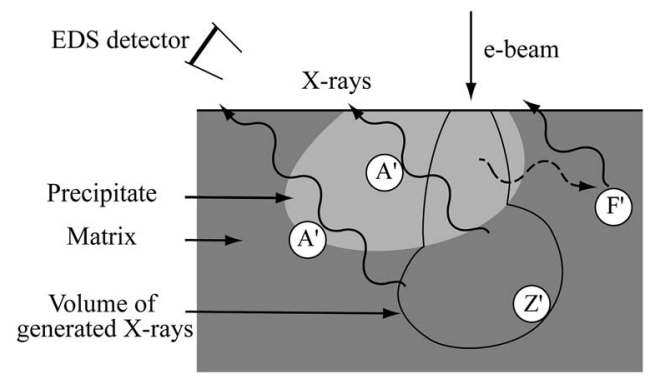

Figure 1. Example of a feature critically small for energy dispersive spectrometry (EDS) analysis. The light gray particle in a darker matrix is smaller than the volume of primary generated $\mathrm{X}$-rays, borders of which are drawn with a black stroke. $\mathrm{Z}^{\prime}, \mathrm{A}^{\prime}$, and $\mathrm{F}^{\prime}$ illustrate the complex matrix effects: $\mathrm{Z}^{\prime}$ the $\mathrm{X}$-ray distributions, $\mathrm{A}^{\prime}$ the absorption along the path to the surface, and $\mathrm{F}^{\prime}$ the fluorescence.

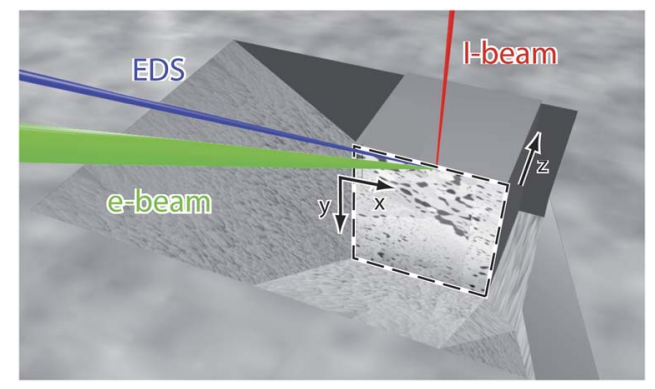

Figure 2. Schematic view of a sample ready for three-dimensional energy dispersive spectrometry (3D EDS) acquisition. The position of the different beams is shown. The sample is tilted to be perpendicular to the ion beam. The freshly milled cross-section that is analyzed is surrounded by a dashed stroke. A protective layer is deposited on the block of interest. The $x, y$, and $z$-axes are defined. The $z$-axis shows the direction of sequential milling.

sample in the case of a known microstructure, such as thin layered films on a substrate (Pouchou \& Pichoir, 1984; Bastin et al., 1993; Merlet, 1995), fibers and particles on a substrate (Small et al., 1978; Statham \& Pawley, 1978) and spheres embedded in a matrix (Gauvin et al., 1995).

The issues in quantifying a spectrum from a heterogeneous and unknown microstructure are illustrated in Figure 1. Examples of the complex effects that need to be compensated for are shown with $Z^{\prime}, A^{\prime}$, and $F^{\prime}: Z^{\prime}$ the $X$-ray generation distribution that varies in a complex way from one material to the other, $\mathrm{A}^{\prime}$ the absorption along the path that goes through different materials to the surface towards the detector, and $\mathrm{F}^{\prime}$ the fluorescence of one material due to X-rays of another material. In order to determine the corrections to compensate for these effects, the sample's microstructure needs to be known in three dimensions. This structure could be estimated from the information provided by mapping. Classical 2D mapping however lacks information in the depth direction, which is particularly important as X-rays tend to be spread more in this primary direction of the entering high-energy electrons. No quantification method has yet been developed for the general case of heterogeneous and unknown microstructure.
This problem can be addressed in 3D EDS microanalysis as the depth information becomes accessible. With voxel size smaller than X-ray depth distribution, the X-rays are emitted from the adjacent voxels, mainly from the deeper ones. With progressive milling of the sample along the depth, the structure influencing the previous analysis is revealed.

The present work introduces a novel "enhanced quantification" method that uses the depth information in order to improve quantification of 3D EDS microanalysis. The method is first described in the light of the existing quantification methods. As a first step to assess the method, three test materials have selected with microstructures of increasing complexity: thin-films, round-shaped particles in a matrix, and a globally complex microstructure. These samples are characterized by $3 \mathrm{D}$ EDS and the acquisition is modeled by Monte Carlo simulations. With the obtained data, enhanced quantification is assessed comparing it to classical quantification and to absolute references.

\section{Materials and Methods}

\section{Existing Quantification Methods}

Quantification for homogeneous samples, referred to here as bulk quantification, is first considered. The elements to be analyzed are identified by their characteristic X-ray lines. For each element, a standard spectrum is measured on a reference sample of known composition under the same experimental conditions. After background correction, $\mathrm{X}$-ray intensities are extracted from both unknown $\left(I_{\text {unk }}\right)$ and standard spectra $\left(I_{\text {std }}\right)$. The content of an element $A$ is approximated with the following $k$-ratio,

$$
k \text {-ratios }{ }_{A}=\frac{I_{\mathrm{unk}, A}}{I_{\mathrm{std}, A}},
$$

$k$-ratios differ from the actual composition as sample and standard have different composition. This difference induces the matrix efects: variation in X-ray ionization $(\mathrm{Z})$ induces variation in X-ray spatial distribution, and absorption (A) and fluorescence (F) differs due to the different elements present. The method to compensate for these effects can be written as

$$
\mathbf{C}=f_{\text {bulk }}(k \text {-ratios }),
$$

where $\mathbf{C}$ is the composition and $k$-ratios are the $k$-ratio of the different elements. The $f_{\text {bulk }}$ used in this work is the well-regarded matrix correction algorithm from Pouchou and Pichoir (1991), the XPP $\varphi(\rho z)$ method.

The XPP $\varphi(\rho z)$ method was extended to the case of thin films on a substrate (Pouchou \& Pichoir, 1993). With an assumption of continuity, the $\varphi(\rho \mathrm{z})$ curves can be derived, and the intensity of one X-ray line emitted in a particular layer can be derived by partial integration. In practice, a layered system is defined with all the a priori knowledge. Guesses are given for the unknown thicknesses and/or compositions. The system is solved through an iterative procedure. $k$-ratios predicted with one system are used to refine the next system. The iteration is stopped when a convergence criteria is reached. In the unfavorable case of 
elements common to different layers, the iteration can converge to a local minimum or may not converge in the limit of the iteration steps. In such cases, $k$-ratios measured at several accelerating voltages should be used.

\section{Enhanced Quantification for 3D EDS Microanalysis}

A step in 3D EDS quantification is schematically plotted in Figure 3. Drawn with dashed boxes, the voxels are indexed with $m, n$ relative to the upper voxel $(i, j)$. The electron beam comes with an angle as the sample surface is tilted in the acquisition geometry used (see Fig. 2). The global shape of $\mathrm{X}$-ray distribution is described with the plotted electron paths. Electrons entered the material with their highest energy in the same direction. After the first scattering events, they start to lose their energy and to diffuse laterally. The highest density of electrons with the highest energy is in a flame in the direction of the electron beam.

Because of the relatively low lateral spreading, the electrons can be regarded as going through a locally homogenous material of composition $\overline{\mathbf{C}}_{j+n}$ in each voxel layer $j+n$. Assuming that layers have a locally uniform composition, equation (2) can be extended to take into account the influence of the deeper layers. The composition of the upper voxel $\left(\mathbf{C}_{i, j}\right)$ is hence given by

$$
\mathbf{C}_{i, j}=f_{\text {layers }}\left(k-\text { ratios }_{i, j}, \overline{\mathbf{C}}_{j+1}, \overline{\mathbf{C}}_{j+2}, \ldots, \overline{\mathbf{C}}_{j+n}, \ldots, \overline{\mathbf{C}}_{j+r_{\max }}\right),
$$

where $r_{\max }$ is the index of the maximum depth of generated $\mathrm{X}$-ray. As a system of uniform layers is equivalent to a stratified sample, the thin-film quantification of Pouchou \& Pichoir (1993) is a candidate for $f_{\text {layers }}$.

To apply equation (3), the voxel composition in two directions needs to be known; after initialization, the equation can be applied recursively through the data in the direction opposite to the milling and in the opposite direction of the $y$-axis. The $k$-ratios of each voxel are corrected for the influence of the subsequent slices. Equation (3) is the recursive relation for which $f_{\text {layers }}$ is the recurrence relation, and the global recursion forms the enhanced-quantification method.

The layer composition $\overline{\mathbf{C}}_{j+n}$ of equation (3) needs to be an accurate approximation of the composition "seen" by the electrons going through the layer. $\overline{\mathbf{C}}_{j+n}$ is defined as the weighted mean of neighboring-voxels in layer $j+n$ :

$$
\mathbf{C}_{j+n}=\sum^{m} \bar{D}_{i, j}(m, n) \mathbf{C}_{i+m, j+n},
$$

where $i$ is the global $y$ index, $m$ the $y$ index relative to $i$, and $\bar{D}_{i, j}(m, n)$ the weighting factors as plotted in Figures 3 for $n=2$. $\bar{D}_{i, j}(m, n)$ can be derived from Monte Carlo simulations. To save computing time, the number of simulations is reduced estimating $\bar{D}_{i, j}(m, n)$ from a limited set of simulations, one per element present in the sample, as follows:

$$
\bar{D}_{i, j}(m, n)=\sum^{A} D_{A}(m, n) k-\operatorname{ratios}_{i, j, A},
$$

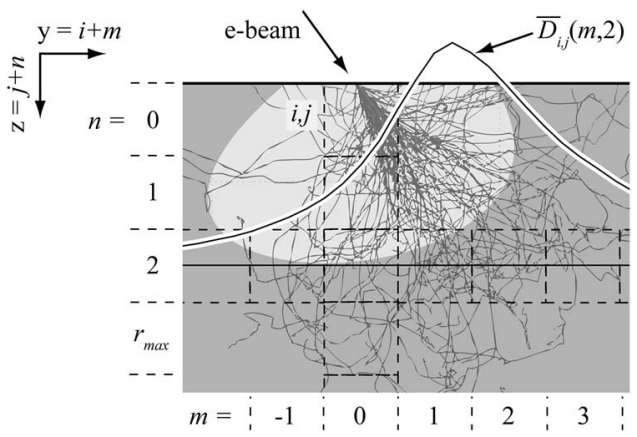

Figure 3. Schematic three-dimensional energy dispersive spectrometry (3D EDS) acquisition of the sample of Figure 1. The electron beam is tilted $36^{\circ}$. A system of voxels is drawn with dashed boxes. An indexing system for $y$ and $z$-axes is defined. The central voxel is indicated by indexes $i, j$ and $m, n$ are indexes relative to this voxel. The plotted electron trajectories are simulated in pure $\mathrm{Al}(5 \mathrm{kV}, 40 \mathrm{~nm}$ voxel size). The corresponding curve of weighting factors $\bar{D}_{i, j(m, n)}$ is plotted in function of continuous $m$ for $n=2$.

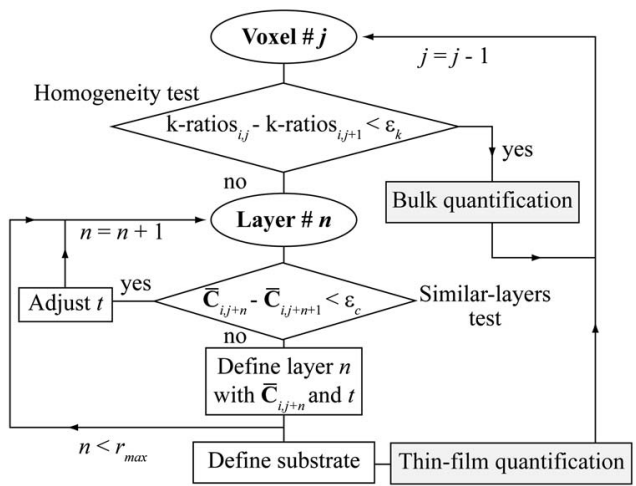

Figure 4. Implementation of the enhanced-quantification recursion. Ovals indicate loops, rectangles indicate actions, and rhombi indicate tests.

where $D_{A}(m, n)$ is the electron distribution in pure material $A$. $D_{A}(m, n)$ gives the number of electrons travelling through a box at the position $y=m$ and $z=n$ and with infinite dimension in $x$-axis. $k$-ratios ${ }_{i, j}$ are taken as an estimation of the global composition.

To use the thin film quantification in equation (3), a system of layers needs to be defined. For a robust recursion, the layer system is simplified at each step. When in homogeneous phase, $k$-ratios of the voxels are similar and bulk quantification is used. When defining the layer system, layers with close compositions are grouped together. In practice, the recursion is implemented as depicted in Figure 4. The main loop on voxel along $j$ goes backwards, toward smaller values of $j$. In the loop, the homogeneity is first tested: if the $k$-ratios of voxels $j$ and $j+1$ are close enough $\left(\sum^{A} \mid k\right.$-ratios $_{j, A}-k$-ratios $\left.\operatorname{rat}_{j+1, A} \mid<\varepsilon_{k}\right)$, the part of the sample under the voxel is considered as homogeneous, and bulk quantification is used. The layer system is then defined. The layers composition $\overline{\mathbf{C}}$ is calculated with equation (4). The $k$-ratios are set as guesses for the unknown composition of 
the upper layer. The layers underneath are defined in a loop along $n$. The similarity between layers is first tested: if compositions of voxels $j+n$ and $j+n+1$ are close enough $\left(\sum^{A}\left|\overline{\mathbf{C}}_{i, j+n, A}-\overline{\mathbf{C}}_{i, j+n+1, A}\right|<\varepsilon_{c}\right)$, they are grouped in one layer and thickness $t$ is adapted. If not, the layer $n$ is defined with a composition $\overline{\mathbf{C}}_{i, j+n}$, and a thickness $t$ corresponding to the voxel size in depth. The loop on $n$ ends when $r_{\max }$ is reached: the substrate is defined with $\overline{\mathbf{C}}_{i, j+r_{\max }}$, and the quantification procedure is applied on the layer system.

All voxels with the same $j$ are calculated before moving to $j-1$. Because $\bar{D}_{i, j}(m, n)$ is shifted toward positive $y$ (see Fig. 3), voxels with larger $i$ are calculated first. When using equation (4) to calculate $\overline{\mathbf{C}}_{i, j+n}$, components of $\mathbf{C}_{i+m, j+n}$ with positive $m$ are thus known. $\mathbf{C}_{i+m, j+n}$ with negative $m$ are approximated by $k$-ratios ${ }_{i+m, j+n}$. Borders in $y$ are extended with mirror voxels. Bulk quantification is used to initialize the recursion at highest value of $j . r_{\max }$ is given by the maximum among all $\mathrm{X}$-ray ranges in pure material calculated with the relation of Anderson \& Hasler (1966). $\varepsilon_{k}$ and $\varepsilon_{c}$ are set typically to 1 wt.\% times the number of elements. The enhanced-quantification method is hence implemented.

\section{Selected Materials}

To properly assess the developed technique with measured data, the samples used need to fulfill some requirements, such as being free of sample-specific artifacts. The selected samples are metallic alloys: they are dense, conductive, well studied and well known, with a microstructure size in the range of the EDS spatial resolution, and with a set of nonoverlapping X-ray lines.

(A) The first selected samples are thin-film deposited on a substrate. This simple structure is suited for a first set of tests. The data here are taken from a database.

(B) The second is an Al- $\mathrm{Zn}$ alloy with a simple microstructure and phase composition, and is thus easy to model.

(C) The third is a weld between nickel-titanium (NiTi) and stainless steel (SS). 3D EDS microanalysis is a suitable characterization technique for the complex microstructure and phase composition of this sample (Burdet et al., 2013).

(A) Thin-film samples were accurately characterized by Bastin \& Heijligers $(2000 a, 2000 b)$. They established a database containing X-ray intensities measured by wave length spectrometry (WDS) on thin films. This database is formed of $k$-ratios from six different thicknesses $(10-320 \mathrm{~nm})$ of $\mathrm{Al}$ and Pd films deposited on 20 different substrates $(Z=4$ to $Z=83)$ and measured at 10 accelerating voltages $(3-20 \mathrm{kV})$. As a WDS detector has a smaller energy resolution than an EDS detector, and as $k$-ratios are independent of acquisition parameters by construction, $k$-ratios of the two techniques are equivalent.

(B) Aluminum is immiscible with Zinc below $277^{\circ} \mathrm{C}$ (see Massalski, 1986) so Al-Zn alloys are composed of two almost pure phases at equilibrium. An Al-Zn alloy was prepared with $78 \mathrm{wt} . \% \mathrm{Zn}$. With a specific heat treatment, the alloy underwent a solid state decomposition and formed a suitable microstructure. The $\mathrm{Al}$ phase is formed in sphere-shaped particles smaller than a micron in a $\mathrm{Zn}$ matrix. The sample used was prepared and was extensively studied by Friedli (2011) in his thesis.

(C) NiTi plays an important role in biomedical engineering thanks to properties such as shape memory and biocompatibility. In order to extend the range of applications, $\mathrm{NiTi}$ is joined with other biocompatible alloys such as SS. During his thesis, Vannod (2011) developed a laser welding technique to join $\mathrm{NiTi}$ wires $(56.4$ wt.\%Ni) and SS (grade $304 \mathrm{~L}$ ) wires, $300 \mu \mathrm{m}$ in diameter. The resulting microstructure is complex in geometry and composition. Phase formation was revealed comparing the phase diagram with the microstructural characterization obtained with 3D EDS by FIB/SEM (Burdet et al., 2013). The same set of data is used here to test the enhanced quantification.

Spatial and compositional references are needed to assure that the obtained compositions accurately describe the actual sample and do not suffer from artifacts. The phase composition of metallic alloys is theoretically predicted at equilibrium in phase diagrams; they are used as compositional references. Due to their low energy, secondary electrons (SE) have an escape depth of less than $\sim 10 \mathrm{~nm}$. As the pixel size of the SE images is small compared with the volume of interaction, the SE generated far from the probe location (SE2) contribute significantly less to the details of the image than the $\mathrm{SE}$ generated at the probe location (SE1). Therefore, the spatial resolution is of the same order of magnitude as the escape depth (see Goldstein et al., 2003). For the studied samples, the escape depth of SE is at least 10 times smaller than the $\mathrm{X}$-ray generation volume. $\mathrm{SE}$ images are consequently used as a spatial reference.

\section{Experimental}

The 3D data of samples (B) and (C) were collected with a Carl Zeiss Nvision40. The angle between the vertical electron column and the ion column (liquid metal ion source, $\mathrm{Ga}^{+}$) was $54^{\circ}$. The InLens detector was used to record the SE images. The EDS detector was an Oxford Instrument X-Max with $80 \mathrm{~mm}^{2}$ detection area. The acquisitions were run with the geometry defined in Figure 2. In this geometry, the milled surface is tilted by $36^{\circ}$ with respect to a surface normal to the electron beam direction. X-rays leave the milled surface toward the detector with a take-off angle of $27.5^{\circ}$. The collection solid angle of the detector is about $0.08 \pm 0.005$ steradians.

Table 1 gives the main acquisition parameters for the two samples characterized by 3D EDS (samples B and C). The accelerating voltage was set to efficiently excite the lowest-energy set of X-ray lines that does not suffer from overlapping peaks, with a detector process time set as low as possible. The probe current was adjusted to obtain 50\% dead time (the detection rate). The acquisition time per map was adjusted to record several maps per hour. The dwell time, the 
Table 1. Acquisition Parameters of the 3D EDS Acquisition.

\begin{tabular}{lcccc}
\hline & $\mathrm{V}_{0}(\mathrm{kV})$ & $\begin{array}{c}\text { Rate } \\
(\mathrm{kct} / \mathrm{s})\end{array}$ & $\begin{array}{c}\text { Dwell } \\
\text { time }(\mathrm{ms})\end{array}$ & $\begin{array}{c}\text { Voxel } \\
\text { size }(\mathrm{nm})\end{array}$ \\
\hline (B) A1-Zn & 5 & 95 & 66 & 40 \\
(C) Weld & 10 & 40 & 29 & 100 \\
\hline
\end{tabular}

$V_{0}$ is the accelerating voltage. "Dwell time" is the time spent to record a spectrum. "Rate" is the count rate (rate of detected X-rays).

$3 \mathrm{D}$ EDS, three-dimensional energy dispersive spectrometry.

time spent to record one spectrum, was accordingly low as given in Table 1. A probe current of 9.2 and $2.8 \mathrm{nA}$ was measured for samples B and $\mathrm{C}$, respectively. The current difference before and after the acquisition was $<1 \%$. The image pixel size was set equal to the slice thickness (isotropic voxel), which was chosen to obtain X-rays emitted from consecutive slices. During each EDS map acquisition, the image shift was compensated by image cross-correlation (SEM images). SE images were recorded with a pixel size eight times smaller than the EDS maps. The standard spectra used to calculate the $k$-ratios were recorded with the same microscope parameters, apart from a much longer acquisition time of $30 \mathrm{~s}$. Pure materials mounted on a separate support were used (Structure Probe Incorporation supplies).

The average number of iterations for the enhanced quantification was about 10 for sample $C$ and less for sample $B$. For sample $\mathrm{C}$, the quantification takes about $3 \mathrm{~ms}$ per voxel on a standard computer (one processor of $3.4 \mathrm{GHz}$ ), a total of $25 \mathrm{~min}$ for the $128 \times 96 \times 44$ voxels. All the data were acquired with the automated solution of Oxford Instruments for 3D EDS. After acquisition, the data were processed with a home-made script. $k$-ratios were extracted using a measured standard with the method described by Goldstein et al. (2003). SEM images were used as a reference to register the whole set of data with the approach developed by Thèvenaz et al. (1998). Quantification (bulk and enhanced) were applied to the set of $k$-ratios using Stratagem library, a commercial software based on the work of Pouchou and Pichoir (1984). Principal component analysis (PCA) was used as a noise filter in the case of the weld sample \& applied to the raw EDS maps with a script developed in the laboratory (Lucas et al., 2013). Before applying the enhanced quantification described before, $k$-ratios were normalized. All Monte Carlo simulations were run with DTSA-II software (Ritchie, 2009). Ten thousand electrons were used to simulate each spectrum of the modeled acquisitions. A hundred thousand electrons were used to simulate the electron distributions for $D_{A}$ (see equation (5)).

\section{Results}

\section{Thin Films: Profile Along $z$}

In a first step, the enhanced quantification is tested on data of the simplest geometry; one dimension profiles characterizing thin-films sample, as described in the upper part of Figure 5.

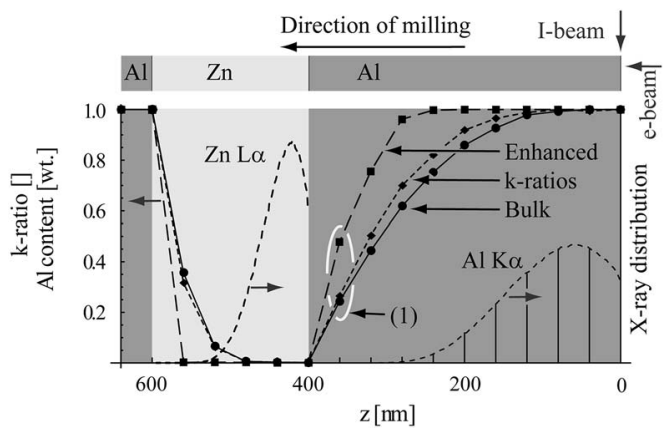

Figure 5. Enhanced quantification applied on a simulated profile along $z$. The thin-film samples and beams position are defined at the top. The profile is simulated at $5 \mathrm{kV}$ with a slice thickness of $40 \mathrm{~nm}$. The enhanced quantification is compared with the bulk quantification and the $k$-ratios (left scale). The two dashed curves give simulated depth distributions of emitted X-ray (right scale). The area under these curves is normalized to one.

The electron beam is perpendicular to the surface, and slices are milled away by the ion beam from right to left. A spectrum is simulated on each subsequent surface and the X-ray intensities are extracted. The resulting data form a profile along $z$ describing the composition of the different layers. A simulated profile along $z$ is shown in the lower part of Figure 5. Experimental profiles along $z$ were extracted from the thin-films database of Bastin \& Heijligers (2000a, $2000 b$ ) considering the film thicknesses as equivalent to the subsequent milled surface.

Simulated data are considered first, then the data from the thin-film database. The lower part of Figure 5 shows a simulated profile through films of pure $\mathrm{Al}$ and pure $\mathrm{Zn}$ with a slice thickness of $40 \mathrm{~nm}$ at $5 \mathrm{keV}$. The dashed curves give the depth distributions of emitted X-ray for the two materials. In both materials, $\mathrm{X}$-rays are emitted from several slices (voxels) up to a maximum of seven slices of $40 \mathrm{~nm}$ in the case of $\mathrm{Al}$ as indicated under the curve. The curve formed by the Al $k$-ratios is consequently deformed close to the interfaces in a shift opposite to the direction of the incident electron beam. The shift is different for the two interfaces as the electrons have to go through a denser or a less dense material first. The range of the influence of the "substrate" (deeper material) is directly related to the X-ray depth distribution. In Figure 5, the recursion of the enhanced quantification goes from left to right. The left interface (AllZn) is perfectly treated; the enhanced quantification returns the actual composition at the first voxel. For the right interface ( $\mathrm{Zn} \mid \mathrm{Al})$, $47 \%$ of $\mathrm{Al}$ is obtained for the first voxel (point (1) in Fig. 5): far from the actual composition, but closer to it than the $k$-ratio. In the next voxel, the layer system is defined with a virtual layer of $47 \%$ of $\mathrm{Al}$ under the upper layer. The result is still not the actual composition, but again closer to it than the $k$-ratio. This continues until the actual composition is reached. The resulting curve forms a sharper interface than the $k$-ratios curve, with an improvement of composition for each voxel up to $25 \%$. The inappropriate bulk quantification gives a slightly worse $\mathrm{Al}$ content compared with $k$-ratios. 


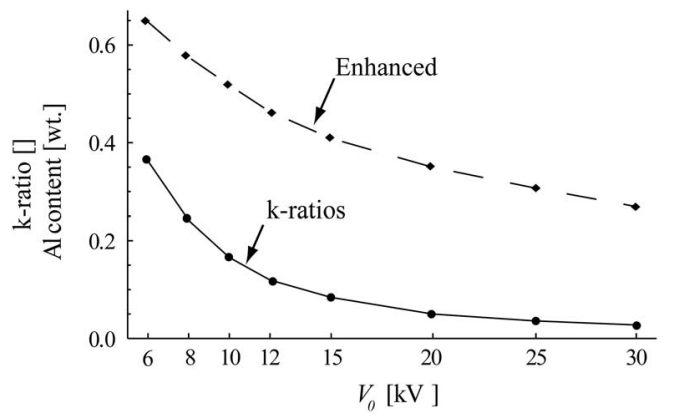

Figure 6. Enhanced quantification applied on profiles extracted from the Al-film database of Bastin \& Heijligers (2000a). Al contents are averaged over the voxels in the $\mathrm{Al}$ film and over the different substrates. The obtained average is plotted as a function of the accelerating voltage $\left(V_{0}\right)$.

Similar results are obtained when applying the enhanced quantification to the experimental $k$-ratios profiles extracted from the thin-films database. Considering the Al thin-films of the database, the corresponding Al-content curves look like the simulated curves of the right interface $(\mathrm{Zn} \mid \mathrm{Al})$ in Figure 5. To extract statistical information over all thin-film systems, $\mathrm{Al}$ content is averaged over all voxels and substrates, and the obtained average is plotted as a function of $V_{0}$ as shown in Figure 6. In this figure, $\mathrm{Al} k$-ratios and $\mathrm{Al}$ contents obtained with enhanced quantification, the input and the output, are compared. A perfectly accurate quantification method would return an average of one pure $\mathrm{Al}$ in every position of the profile. The $k$-ratios and enhancedquantification curves similarly increase, as $V_{0}$ decreases. When $V_{0}$ is lower more $\mathrm{X}$-rays are emitted from the film, and the profiles form a sharper interface for the $\mathrm{Al} k$-ratios and hence for the enhanced $\mathrm{Al}$ contents. The difference between curves is relatively constant, around $30 \%$. $V_{0}$ is the parameter best showing the correlation between inputs and outputs.

The accuracy of enhanced quantification does not only depend on $k$-ratios as seen with one other parameter: the maximum range of substrate X-ray line in substrate $\left(R_{x, \text { sub }}\right)$ for a fixed $V_{0} . R_{x, \text { sub }}$ is calculated with the relation of Anderson \& Hasler (1966); it depends on the substrate density, $V_{0}$, and the $\mathrm{X}$-ray energy. In Figure 7 , the $\mathrm{Al}$ content is averaged over all voxels and $V_{0}$, and the obtained average is plotted in function of $R_{x, \text { sub }}$ relative to $R_{x, \text { film }}$ (range of $\mathrm{Al} \mathrm{K \alpha}$ in bulk $\mathrm{Al}$ ). The higher $R_{x, \text { sub }}$ is, the higher is the enhancement in $\mathrm{Al}$ content, while $k$-ratios show little variation and no clear correlation. The vertical dashed line gives $R_{x, \text { sub }}$ for three substrates of various density. The enhancement is one of the lowest on an $\mathrm{Au}$ substrate and is medium on a $\mathrm{Zr}$ substrate. The best result is obtained with a $\mathrm{Si}$ substrate that has $R_{x, \text { sub }} / R_{x, \text { film }}$ higher than one. Results on Pd films are globally better, and the same behavior is observed. The curve of the enhanced quantification quickly reaches one with $R_{x, \text { sub }} / R_{x, \text { film }}$ higher than one. The fact that $k$-ratios are not influenced by $R_{x \text {,sub }}$ suggests that the observed behavior is induced by inaccuracies of the enhanced quantification and, consequently, of the thin-film quantification.

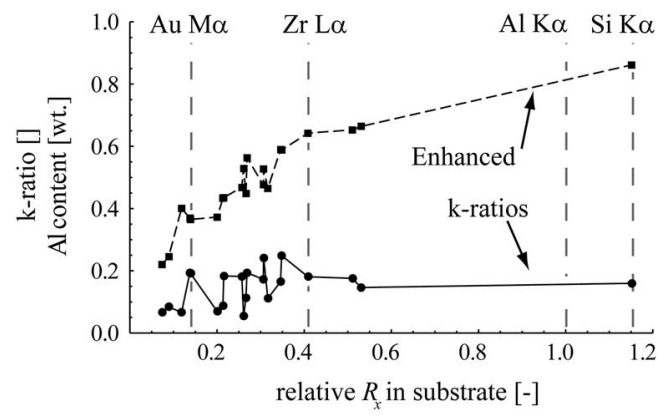

Figure 7. Enhanced quantification applied on profiles extracted from the Al-film database of Bastin \& Heijligers (2000a). Al contents are averaged over the voxels in the $\mathrm{Al}$ film and over the different accelerating voltages $\left(V_{0}\right)$. The obtained average is plotted as a function of the maximum range of substrate X-ray line in substrate $\left(R_{x, \text { sub }}\right)$ at a fixed $V_{0} . R_{x, \text { sub }}$ is scaled relatively to $R_{x, \text { film }}$ the range of $\mathrm{Al} \mathrm{K} \alpha$ in bulk $\mathrm{Al}$.

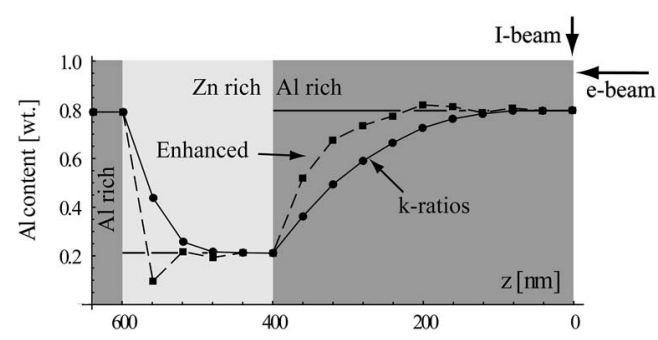

Figure 8. Enhanced quantification applied on a simulated profile along $z$ through compound materials. The profile is simulated at $5 \mathrm{kV}$ with a slice thickness of $40 \mathrm{~nm}$. The $\mathrm{Al}$ rich phase contains 20 wt.\%Zn, and the $\mathrm{Zn}$ rich phase contains 20 wt.\%Al. Actual Al content is indicated by horizontal grey lines. Interfaces are parallel to the surface situated at right. The beams position is indicated at the top-right corner.

To consider a more complex case, a profile through compound materials containing the same elements is simulated. The sample is formed of an Al-rich phase containing $20 \mathrm{wt} . \%$ $\mathrm{Zn}$ and a $\mathrm{Zn}$-rich phase containing $20 \mathrm{wt} . \% \mathrm{Al}$, as shown in Figure 8 . The profile along $z$ is simulated with the same parameters used in Figure 5. In Figure 8, the enhanced $\mathrm{Al}$ contents are globally closer to the actual composition than bulk quantification. However, a dampened oscillation is observed for the left interface. The first voxel after the interface has an $\mathrm{Al}$ content $10.7 \%$ too low, the next has an $\mathrm{Al}$ content $1.4 \%$ too high, and the following has an $\mathrm{Al}$ content $1.0 \%$ too low. The oscillations are stopped when the homogeneity test is fulfilled and the bulk quantification is used. This behavior is less pronounced for the other interface. The oscillations are due to inaccuracies of the thin-film quantification at one voxel reported on the following one during the recursion.

\section{Al-Zn Alloy: Round-Shaped Particles}

In a next step, the enhanced quantification is tested on roundshaped particles of the Al-Zn sample. Before considering the 
measured data, a data set is simulated on a model sample formed by a sphere of pure $\mathrm{Al}$ in a pure $\mathrm{Zn}$ matrix. The sphere has a diameter of $280 \mathrm{~nm}$, slightly smaller than the range of $\mathrm{Al}$ $\mathrm{K} \alpha$ in $\mathrm{Al}(\sim 300 \mathrm{~nm})$. The sample is mapped with $11 \times 11 \times 14$ voxels. Orthogonal views through the sphere center are shown in Figure 9. Inside the sphere, the highest $\mathrm{Al} k$-ratio is 0.75 ; a good amount of $\mathrm{Zn} \mathrm{L} \alpha \mathrm{X}$-rays are emitted from the matrix. $\mathrm{Al}$ content is far from $100 \%$ for the bulk quantification, but it is about 30\% higher for the enhanced quantification. Outside the sphere, the Al content is generally lower for the enhanced quantification. In the same way as with profiles along $z$, a shift is observed opposite to the electron beam direction (along the $y$ and $z$ directions). This shift is partially corrected with the enhanced quantification.

Figure 10 shows orthogonal views through the stack of experimental data. Particle shapes are well defined in the SE stack. The volume of interest contains some larger particles of $\sim 300 \mathrm{~nm}$ at a low value of $z$. The smaller particles have an average size of $\sim 100 \mathrm{~nm}$. In the bulk-quantified stack, particles appear more blurry than in the enhanced-quantified stack.
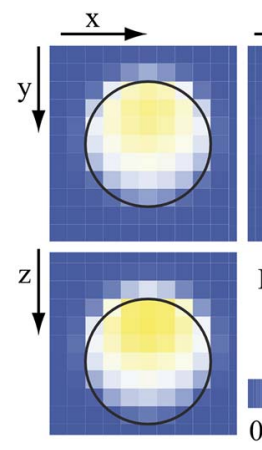
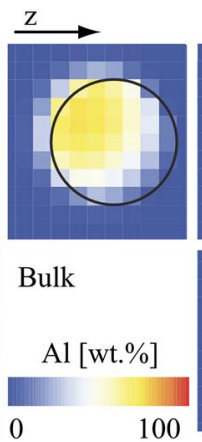
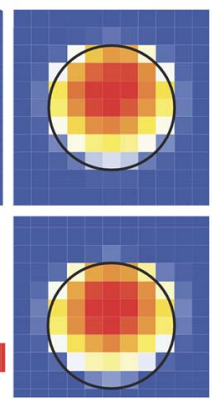

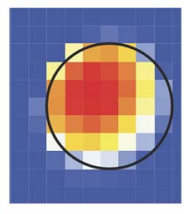

Enhanced
Figure 9. Enhanced quantification applied on simulated $3 \mathrm{D}$ acquisition. Modeling the Al- $\mathrm{Zn}$ sample, the simulated sample is composed of an $\mathrm{Al}$ spherical particle of $280 \mathrm{~nm}$ in a $\mathrm{Zn}$ matrix. The acquisition is simulated at $5 \mathrm{kV}$ with voxel size $40 \mathrm{~nm}$ in all directions. The colored maps give the $\mathrm{Al}$ content of orthogonal views through the sphere center. The sphere border is drawn with black circles.
In the latter stack, higher $\mathrm{Al}$ concentration is obtained at the particle positions given by the SE image. Inside the bigger particles, the enhanced-quantified maps show an uneven pattern similar to noise for $y z$ and $x z$ maps, but less marked for the $x y$ map at the top left.

Figure 11 gives a closer look at this artifact, with a profile along $z$ through the big particle at the crosshair position in Figure 10 . The $k$-ratios and the enhanced quantification, the input and the output, are compared. The SE signal gives a spatial reference for the position of the interfaces. At the right interface, the three curves have a sharp and regular shape and enhanced content is lower than the $k$-ratio forming a curve close to the SE reference. At the left interface, the $k$-ratio curve is flatter and has a slightly irregular shape. The enhanced content is higher overall than the $k$-ratio, but the curve oscillates between voxels. A $k$-ratio with a value slightly higher than the mean slope results in $\mathrm{Al}$ content clearly higher than the mean slope [e.g. point (1)], overcompensated in the next step [e.g. point (2)]. This behavior is linked to inaccuracies passed on by the recursion, similar to what is observed for compound materials (see Fig. 8). However in this case, inaccuracies are mainly due to noise in the input data. Smoothing the input $k$-ratios, the oscillations of the enhanced curve is reduced. The enhanced quantification hence amplifies the noise. The recursion is along $z$, oscillations are thus observed only in this direction. As oscillations have a short period they can be easily smoothed, for instance with a mean filter along $z$.

\section{NiTi-SS Weld: Complex Structure}

In a final test, the enhanced quantification is tested on the complex microstructure of the NiTi-SS sample. Before considering the measured data, an acquisition is simulated with profiles along $z$ with a low level of noise. Figure 12 shows a profile between $\gamma$ - $(\mathrm{Fe}, \mathrm{Ni})$ and $\mathrm{Fe}_{2} \mathrm{Ti}$, two phases that compose one of the fine microstructures of the sample. The phase composition is approximated based on the quantified maps and the Ti-Fe-Ni phase diagram (from Cacciamani et al., 2006).
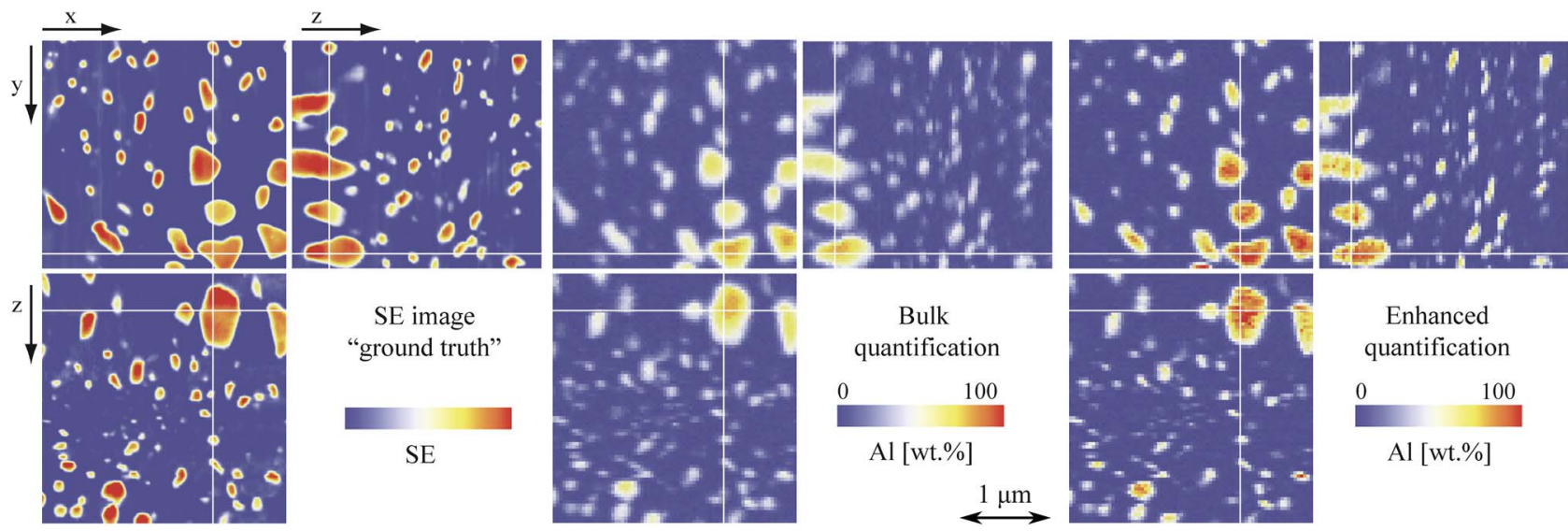

Figure 10. Enhanced quantification applied on data from the Al-Zn-sample acquisition. Orthogonal views through the stack of SE images (ground truth), bulk and enhanced quantification are shown. The color scale of SE images is set arbitrarily to obtain pictures similar to the others. The white lines show the position of the other orthogonal views. 


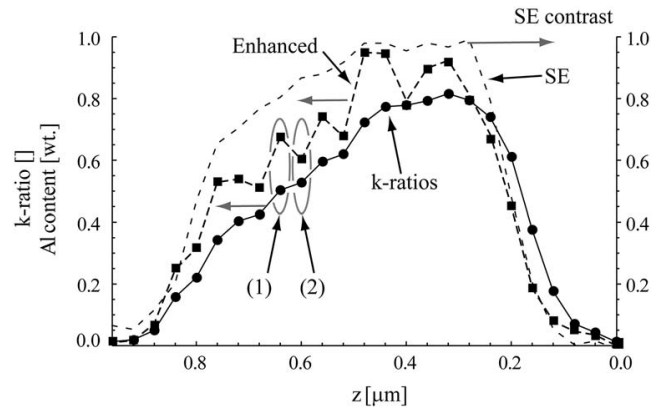

Figure 11. Details on the measured data of the Al- $\mathrm{Zn}$ sample, showing the oscillating behavior of the enhanced quantification. Profile along $z$ through the big particle at the crossing of the white lines of Figure 10. SE contrast is adjusted from 1 to 0.

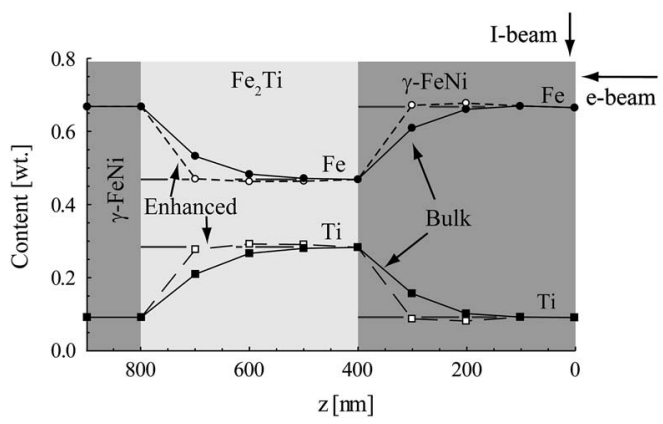

Figure 12. Enhanced quantification applied on a simulated profile along $z$ through two phases of the weld sample, $\gamma$-(Fe,Ni) and $\mathrm{Fe}_{2} \mathrm{Ti}$ phases. The profile is simulated at $101 \mathrm{kV}$ with a slice thickness of $100 \mathrm{~nm}$. Ni content is constant and not displayed. The phase composition is indicated by horizontal grey lines. The interfaces are parallel to the surface situated at right. The beam position is indicated at the top-right corner.

The highest X-ray range $\left(R_{x}\right)$ is $390 \mathrm{~nm}$ in $\gamma$ - $(\mathrm{Fe}, \mathrm{Ni})$ and $430 \mathrm{~nm}$ in $\mathrm{Fe}_{2} \mathrm{Ti}$, which corresponds to 4.3 times the voxel size (7.75 for Al-Zn sample). The Ni content is constant and equal in the two phases and is not displayed. The two closest voxels at the right of the interfaces deviate from the phase composition and are corrected by the enhanced quantification. Because of the short range of influence, the oscillation problem observed for Al-Zn compounds (see Fig. 8) is strongly reduced in this profile.

Dwell time and acquisition rate are lower than for the Al-Zn sample (see Table 1). A spectrum acquired on the weld sample contains only 1,000 counts as plotted in gray in Figure 13. It is noisy and so is the corresponding Ti-content map on the left (bulk quantification). When applying the enhanced quantification on such noisy data, the increase in noise induced by the method hides all possible gains. Noise reduction becomes indispensable. Using the PCA, the raw set of spectra is decomposed and reconstructed using only the most meaningful components, leaving apart the noise (see Lucas et al., 2013). The reconstructed spectrum is strongly smoothed, as observed in Figure 13. The main peaks are efficiently fitted, as confirmed with the smoothed Ti-content map on the right: features that were unseen are

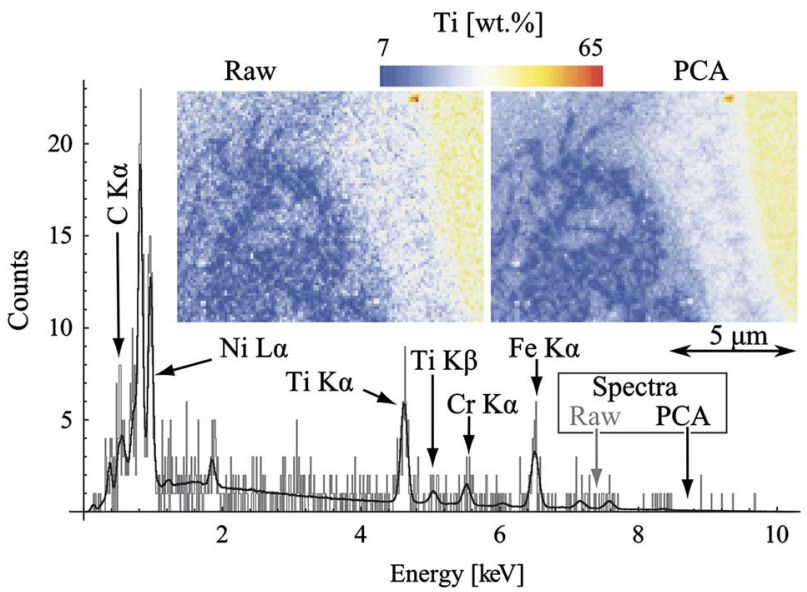

Figure 13. Principal component analysis (PCA) used as a noise filter and applied on the raw set of spectra from the weld-sample acquisition (experimental parameters given in Table 1). A raw spectrum is plotted in gray, and its reconstruction after PCA decomposition is plotted in black. The set of X-ray lines used for quantification and $\mathrm{Ti} \mathrm{K} \beta$ is indicated. Scaled by a color code, the Ti-content maps are obtained by bulk-quantifying the raw spectra at left and the reconstructed spectra at right.

revealed and can be correlated to details of the SE image of Figure 14. The reconstructed spectrum clearly shows small peaks, such as $\mathrm{Ti} K \beta$, that are buried in the noise in the raw spectrum. These peaks should not be used for quantification as they are likely to be correlated to other peaks with higher signal-to-noise ratio, such as $\mathrm{Ti} \mathrm{K} \alpha$ in the case of $\mathrm{Ti} \mathrm{K} \beta$. PCA does not remove the requirement of a high enough signal-to-noise ratio for a reliable quantitative analysis.

Before applying any quantification, the raw set of spectra is treated by PCA. Bulk and enhanced quantification is then applied and displayed in Figure 14. In this figure, orthogonal views through the stacks of SE images and of quantified $\mathrm{Ti}$ contents are shown. The Ti content is considered because it is different in each phase. In Figure 15, the $\mathrm{Fe}-, \mathrm{Ni}-$, and Ti-concentrations measured at each voxel of the quantified 3D stacks are reported in a ternary Gibbs simplex, with a logarithmic scale (from blue to red). This type of representation, called here ternary histogram, was suggested by Bright \& Newbury (1991). In Figure 15, they are overlaid on a $1,000^{\circ} \mathrm{C}$-isothermal section of the $\mathrm{Fe}-\mathrm{Ni}$ - $\mathrm{Ti}$ phase diagram (Cacciamani et al., 2006).

The enhanced quantification clearly increases the contrast in the Ti maps, especially in regions with fine microstructure. On the other hand, the enhanced quantification increases the noise, still present after smoothing with PCA, as can be observed in the Ti maps and in the peak broadening in the ternary histogram. The features revealed by the enhanced contrast in Ti content can be linked to the fine details of the SE image, for instance in the region indicated by the red area of the inset at the bottom right of the SE images in Figure 14. This region is formed by $\gamma-(\mathrm{Fe}, \mathrm{Ni})$ and $\mathrm{Fe}_{2} \mathrm{Ti}$ phases, the same phases used for the simulated profile of Figure 12. In this profile, voxels close to the interface show an increase/decrease 

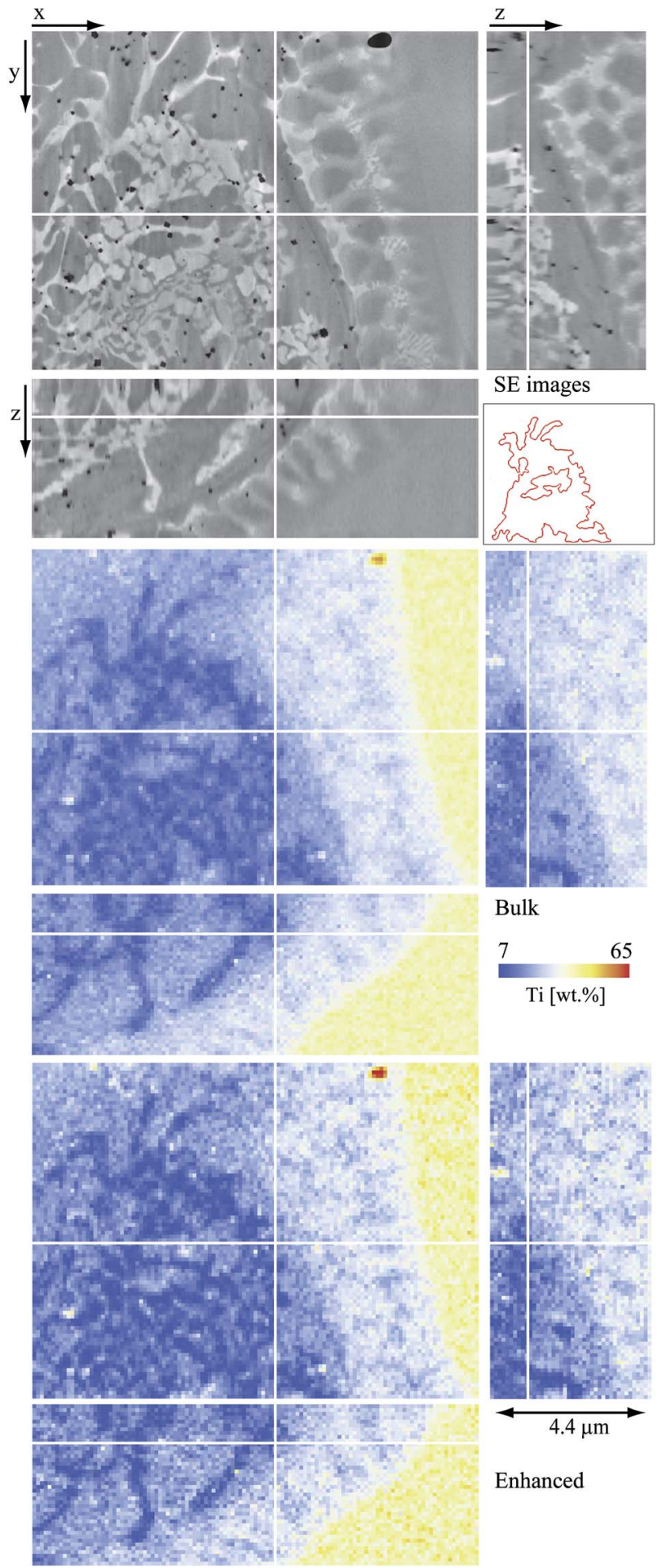

Enhanced

Figure 14. Enhanced quantification applied on data from the weld-sample acquisition. Orthogonal views through the stack of SE images, bulk, and enhanced quantification are shown. Both quantifications are preceded by principal component analysis (PCA) noise filtering. The white lines show the position of the other orthogonal views. In the inset at the bottom right of the SE images, the area drawn in red corresponds to the fine microstructure formed by $\gamma$-(Fe,Ni) and $\mathrm{Fe}_{2}$ Ti phases.



Figure 15. Ti-Fe-Ni ternary histograms characterizing the whole set of quantified composition from the weld sample. A ternary phase diagram cut at $1,000^{\circ} \mathrm{C}$ is superimposed (from Cacciamani et al., 2006). The bulk quantified stack is compared with the enhanced quantified stack. Both quantifications are preceded by principal component analysis (PCA) filtering.

of $\mathrm{Ti}$, being in a phase rich/poor in $\mathrm{Ti}$, respectively. This corresponds perfectly to the observed increase in Ti contrast. Similarly predicted by simulations, the enhanced Fe stack shows an increased contrast, while the contrast of the $\mathrm{Ni}$ stack stays constant. In the ternary histogram of Figure 15, this should correspond to a further split of the $\gamma$ - $(\mathrm{Fe}, \mathrm{Ni})$ and $\mathrm{Fe}_{2} \mathrm{Ti}$ peaks along a line of constant $\mathrm{Ni}$. The $\gamma$ - $(\mathrm{Fe}, \mathrm{Ni})$ peak indeed moves to higher values of $\mathrm{Fe}$, closer to the composition predicted by the phase diagram. However, the shift of $\mathrm{Fe}_{2} \mathrm{Ti}$ peak is not significant, since the $\mathrm{Fe}_{2} \mathrm{Ti}$ phase mainly forms a coarser microstructure that is less influenced by the enhanced quantification.

\section{DiscusSION}

For all simulated and measured data, the developed enhanced quantification significantly improves the accuracy of composition compared with the well-established bulk quantification. Quantified compositions are closer to the actual compositions, which are known or predicted based on phase diagrams. A gain of about 25 wt.\% is measured over the wide range of elements of Bastin's database. For the two sets of measured data, this improved accuracy results in a better resolution of fine microstructures. The interaction volume induces a shift opposite to the direction of the incident electron beam. This shift is partially corrected with the enhanced quantification. The phase boundaries in the EDS maps are closer to the interfaces observed in the SE images, which are known or approximated with the SE images.

Applying enhanced quantification to the different data, the benefits of the method are established, as well as its weak points. In this part, these weak points are discussed exploring different possibilities for further improvements. The most noticeable one is an oscillating behavior induced by the recursive nature of the method. In a recursion, imprecisions of one step impacts on the next steps, and the fluctuations due to imprecisions are amplified. Considering the spatial evolution of composition, the sharper it is, the smoother it gets, as the gradient is higher than the imprecisions on the 
composition. Sharper variations hence show less oscillation after the enhanced quantification, as can be clearly observed for the Al-Zn sample. The presented method was consequently modified applying a cut-off for a compositional gradient that is too low with a "homogeneity test". If two sets of subsequent $k$-ratios in the main recursion are close enough, the bulk quantification is used. This simple test greatly helps to reduce oscillations, but does not differentiate noise from actual compositional gradient due to its local nature and can be subject to improvement. The oscillations can also be reduced afterwards as they have specific characteristics: a short period only along $z$. A mean filter in this direction is appropriate and shows good results. Part of the enhanced quantification gain is however lost, regardless of the filter.

The imprecisions amplified by the recursion are either present in the experimental input, or generated by the processing of each recursion step. On the experimental side, the noise is clearly the highest source of imprecision as a short time needs to be spent per spectrum. To minimize the noise, the acquisition rate is maximized reducing the energy resolution to the minimum required and using the highest electron beam current possible. Despite that, the spectra of the weld sample are particularly noisy, and noise filtering, such as the PCA, is a necessary step before the enhanced quantification. PCA, a multivariate statistical technique, provides highly effective noise-reduction accounting for the numerous and correlated spectra that form the stack. Using PCA as a noise filter is, however, a non-standard technique that can suffer from artifacts if the considered peaks have a low signal-to-noise ratio. It should be used with care and requires visual inspection of the reconstructed spectra, as further discussed for the weld-sample data by Lucas et al. (2013) and by Burdet (2012, chap. 5).

Apart from the noise, other potential sources of experimental imprecision are the parasite $\mathrm{X}$-rays and the drift. The parasite $\mathrm{X}$-rays are generated in the surrounding wall, and their influence can be considered a global bias of the input, as observed by Burdet (2012, chap. 4). They do not add fluctuation at a voxel length scale and thus have a reduced influence on the oscillation problem. This unwanted signal can be suppressed with the acquisition geometry suggested by Schaffer \& Wagner (2008), not used here because it is more time consuming. The drift is minimized with a carefully stabilized microscope and no stage movement. On the same microscope, acquisitions with smaller slice thickness showed minimal drift ( Cantoni et al., 2010). During EDS map acquisition, image shift is compensated by image correlation (SEM image). After acquisition, the remaining shift between subsequent images is reduced applying on all stacks the same registration obtained with the SE images, which have pixel size eight times smaller than the EDS maps. However, the imprecision on slice thickness remains unknown. It is more likely to show variation at a longer period than a single slice thickness. In a further improvement, the slice thickness can be measured and used as an input of the enhanced quantification.
The precision of the processing can be evaluated by considering the recursion step in two parts: the system of layers is established and the X-ray depth distributions ( $\varphi(\rho \mathrm{z})$ curves) are determined. In the first part, the X-ray spreading due to the tilt is taken into account. The different compositions seen by the electrons in a particular layer are approximated by an average composition. The average is weighted following electron distributions, which are simulated in pure elements before the recursion. This rather straight approximation can be improved with more realistic simulations, but at the expense of computing time. The second step of determining the $\varphi(\rho \mathrm{z})$ curves is extensively tested with the profiles along $z$, for which the noise is low and the layers have a constant composition. Lower performances are observed and can be linked to two unfavorable cases of thin-film quantification described by Pouchou (2002). When the ratio between X-ray ranges of film and substrate $\left(R_{x, \text { sub }} / R_{x, \text { film }}\right)$ is high, the main assumption of the thin-film quantification, the continuity of $\varphi(\rho \mathrm{z})$ curves, is more likely to be invalidated as observed with Bastin's database. When different layers share the same elements, the $\varphi(\rho z)$ curves become hard to determine unambiguously, resulting in a local solution. In the presented method, the most unfavorable layer systems are avoided by grouping together layers of similar composition. Lower performances are still observed in critical cases, such as profiles through compound materials containing the same elements. In such samples, oscillations can be reduced by a small enough ratio between the maximum X-ray range and the slice thickness $\left(r_{\max }\right)$. Otherwise, ambiguous systems are more likely to be resolved using all available high and low energy X-ray lines, as stressed by Pouchou (2002). However, careful work needs to be done on peak deconvolution as low energy peaks have more chance to overlap.

For further progress, the method needs to be modified more extensively. To reduce imprecisions of the thin-film quantification, its accuracy can be predicted with the method developed by Statham (2010) and can be used to weigh each step of the recursion. To increase the precision, the enhanced quantification can be applied iteratively aiming for a solution with the lowest total variation. To correct the matrix-effects (absorption and fluorescence) more accurately, the X-ray path to the surface toward the detector can be considered as a succession of voxels, and not as a succession of layers like in the present method. Acting at different levels, these improvements are believed to greatly improve the enhanced quantification. The oscillation could be reduced to the level of the input imprecisions while keeping or increasing the gain obtained with the presented method.

\section{CONCLUSIONS}

In this paper, a method to correct 3D EDS data for effects of the large volume of X-ray emission was presented. The method is recursive along the $z$ direction and backwards from the depth to the surface. At each step of the recursion, the influence of the deeper neighboring voxels is corrected. In an approximation of the actual sample, a layers system is defined, on which thin-film quantification is applied to obtain the 
corrected composition. This enhanced quantification was applied on data from a thin-film database, from simulations, and from acquisitions on two metallic alloys. The method showed clear improvement of the composition accuracy for all tested data compared with classical (bulk) quantification. The shift induced by the emission volume is partially corrected, interfaces appear sharper, and small features are more clearly revealed. The concept of the method is thus proven.

Out of the different application examples, the weak points of the method were identified. The main artifact is an oscillating behavior induced by the recursion that amplifies the imprecisions. The experimental and processing imprecisions are reduced optimizing the acquisition and the recursion implementation, respectively. On the experimental side, noise and drift are minimized. On the processing side, the most unfavorable cases for thin-film quantification are avoided, the noise is reduced with a multivariate statistical technique, and the drift between subsequent EDS maps is carefully corrected.

In the discussion of the imprecision sources, different possibilities of improvements were identified. Some weaker points can be optimized, for instance using measured slice thicknesses as an input of the method or defining each layer system with a more accurate model. The method can be modified more extensively, for instance applying it iteratively in a way to converge to a result with reduced oscillations. The presented improvements are believed to largely improve the technique and will be explored in future work.

\section{ACKNOWLEDGMENTS}

This work is the result of a four-year project thesis financed by Carl Zeiss. Thanks to them and to Oxford Instrument for technical support. Thanks to Guillaume Lucas for his help with PCA and for helpful conversation. Thanks to Jonas Vannod and to Jonathan Friedli for providing the sample. Thanks to Paul Midgley, Patrick Hoffmann, and Thierry Epicier for fruitful discussion during and after the thesis exam.

\section{Associated}

Additional information can be found in the author thesis freely accessible under http://infoscience.epfl.ch/record/ 181656 (Burdet, 2012).

\section{REFERENCES}

Anderson, C.A. \& Hasler, M. (1966). X-ray range. In Proccedings of the 4th International Conference on X-ray otpics and Microanalysis, Castaing R., Deschamps P. \& Philibert J. (Eds.), pp. 310. Paris: Wiley.

Bastin, G.F., Dijkstra, J.M., Heijligers, H.J.M. \& Klepper, D. (1993). In depth profiling with the electron probe microanalyzer. Microbeam Anal 2, 29-43.

Bastin, G. \& Heijligers, H. (2000a). A systematic database of thin-film measurements by EPMA: part I-aluminum films. $X$ Ray Spectrom 29, 212-238.
Bastin, G. \& Heijligers, H. (2000b). A systematic database of thin-film measurements by EPMA: part II-palladium films. $X$ Ray Spectrom 29, 373-397.

Bright, D. \& Newbury, D. (1991). Concentration histogram imaging: A scatter diagram technique for viewing two or three related images. Anal Chem 63, 243A-250A.

Burdet, P. (2012). Three dimensional microanalysis by energy dispersive spectrometry: Improved data processing. Ph.D. thesis, Ecole Polytechnique Fédérale de Lausanne, Lausanne.

Burdet, P., Vannod, J., Hessler-Wyser, A., Rappaz, M. \& Cantoni, M. (2013). 3D chemical anaylsis of laser welded NiTi: Stainless steel wire using a dualbeam FIB. Acta Mater 61(8), 3090-3098.

Cacciamani, G., De Keyzer, J., Ferro, R., Klotz, U., Lacaze, J. \& Wollants, P. (2006). Critical evaluation of the Fe-Ni, Fe-Ti and Fe-Ni-Ti alloy systems. Intermetallics 14, 1312-1325.

Cantoni, M., Genoud, C., Hébert, C. \& Knott, G. (2010). Large volume, isotropic, 3D imaging of cell structure on the nanometer scale. Microsc Anal 24, 13-16.

FriedLI, J. (2011). Interfacial energy anisotropy and growth morphologies in aluminium-zinc Alloys. Ph.D. thesis, Ecole Polytechnique Fédérale de Lausanne, Lausanne.

Friel, J. \& Lyman, C. (2006). Tutorial review: X-ray mapping in electron-beam instruments. Microsc Microanal 12, 2-25.

Gauvin, R., Hovington, P. \& Drouin, D. (1995). Quantification of spherical inclusions in the scanning electron microscope using Monte Carlo simulations. Scanning 17, 202-219.

Goldstein, J., Newbury, D.E., Echlin, P., Joy, D.C., Lyman, C.E., Lifshin, E. \& SAwyer, L. (2003). Scanning Electron Microscopy and X-Ray Microanalysis. New York: Plenum Publishers.

Lucas, G., Burdet, P., Cantoni, M. \& Hébert, C. (2013). Multivariate statistical analysis as a tool for the segmentation of $3 \mathrm{D}$ hyperspectral data. Micron 52, 49-56.

Massalski, T.B. (Ed.) (1986). Chapter Al-Zn. In Binary alloy Phase Diagrams: Volume 1 (Ac-Au to Fe-Rh), pp. 184-188. Ohio: American Society for Metals.

MERLET, C. (1995). A new quantitative procedure for stratified samples in EPMA. Proceedings of the 29th Annual Conference of the Microbeam Analysis Society, Etz E.S. (Ed.), pp. 203-204. New York: VHC.

Pouchou, J. \& Pichorr, F. (1984). New model for quantitative $\mathrm{X}$-ray microanalysis. part II: Application to in-depth analysis of heterogeneous samples. Rech Aerospatiale 5, 47-65.

Pouchou, J.L. \& Pichoir, F. (1991). Quantitative analysis of homogeneous or stratified microvolumes applying the model PAP. In Electron Probe Quantitation, Heinrich K.F.J. \& Newbury D.E. (Ed.), pp. 31-75. New York: Plenum Press.

Pouchou, J.L. \& Pichoir, F. (1993). Electron probe X-ray microanalysis applied to thin surface films and stratified specimens. Scanning Microsc Suppl 7, 167-189.

Pouchou, J.-L. (2002). X-ray microanalysis of thin surface films and coatings. Mikrochim Acta 138, 133-152.

Ritchie, N.W. (2009). Spectrum simulation in DTSA-II. Microsc Microanal 15, 454-468.

SCHAFFER, M. \& WAGNER, J. (2008). Block lift-out sample preparation for $3 \mathrm{D}$ experiments in a dual beam focused ion beam microscope. Microchimica Acta 161, 421-425.

Schaffer, M., Wagner, J., Schaffer, B., Schmied, M. \& Mulders, H. (2007). Automated three-dimensional x-ray analysis using a dual-beam FIB. Ultramicroscopy 107, 587-597.

Small, J.A., Heinrich, K.F.J., Fiori, C.E., Myklebust, R.L., Newbury, D.E. \& Dillmore, M.F. (1978). The production and characterization of glass fibers and spheres for microanalysis. Scanning Electron Microsc I, 445-454. 
Statham, P.J. \& PAwley, J.B. (1978). New method for particle X-ray Micro-analysis based on peak to background measurements. Scanning Electron Microsc I, 469-478.

Statham, P.J. (2010). Feasibility of x-ray analysis of multi-layer thin films at a single beam voltage. IOP Conf Ser: Mater Sci Eng 7, 012027.
Thèvenaz, P., Ruttimann, U. \& Unser, M. (1998). A pyramid approach to subpixel registration based on intensity. IEEE $T$ Image Process 7, 27-41.

VANNOD, J. (2011). Laser welding of nickel-titanium and stainless steel wires: Processing, metallurgy and properties. Ph.D. thesis, Ecole Polytechnique Fédérale de Lausanne, Lausanne. 